\title{
Safety and tolerability of virucidal hand rubs: a randomized, double-blind, cross-over trial
}

\author{
A Conrad ${ }^{1}$, D Cosic ${ }^{1}$, C Schmoor $^{2}$, M Dettenkofer $^{1^{*}}$ \\ From International Conference on Prevention \& Infection Control (ICPIC 2011) \\ Geneva, Switzerland. 29 June - 2 July 2011
}

\section{Introduction / objectives}

Clinical trial to evaluate the safety and tolerability of different virucidal hand rubs.

\section{Methods}

In a randomized, double-blind, 4-period cross-over trial, healthy volunteers received 3 different virucidal hand rubs (P1-3) and a reference product $(\mathrm{R})$ in a randomized sequence each over a period of 4 days $(90 \mathrm{~mL} / \mathrm{d})$ with a wash-out period of 10 days. Primary endpoint was skin barrier function measured by transepidermal water loss (TEWL) in $\mathrm{g} / \mathrm{hm}^{2}$ at the end of the 4-day application period. Secondary endpoints were corneometry, skin status score, and adverse events (AE). TEWL and corneometry were analyzed in linear models. The effects were estimated as differences between agents with $95 \% \mathrm{CI}$ and tested with a 2-sided level of 0.05 .

\section{Results}

22 subjects (7 males, 15 females) were randomized and started at least one period. TEWL was $22.5,95 \%>C I$ [19.6,25.4], 16.3 [13.5,19.1], 16.4 [13.4,19.3], and 24.0 $[21.1,27.0]$ after P1, P2, P3, and R; $\mathrm{p}<0.0001$. Corneometry was 49.7 [40.7,58.6], 45.4 [36.9,53.9], 64.3 [55.3,73.2], and 48.9 [39.6,58.1] after P1, P2, P3, R; $\mathrm{p}=0.005$. The median skin status score dorsal was $0.5,0,1,0.5$ after P1, P2, P3, R. The percentage of subjects who experienced at least one $\mathrm{AE}$ was $86 \%, 25 \%, 89 \%$, and $56 \%$ with P1, P2, P3, R. The majority of AEs were skin reactions (no serious AEs).

\section{Conclusion}

Results were inconsistent, e.g. P3 showed low TEWL (= fair skin barrier function) but high skin status score and high number of adverse events (= impaired tolerability). Assessment of TEWL may be influenced by non volatile compounds in this setting and not be a suitable measure. Number of AEs was higher in products containing phosphoric acid (P1, P3). In general, number of AEs was higher than expected for all products requiring further improvement in hand rub development.

\section{Disclosure of interest}

A. Conrad Grant/Research support from B.Braun, Melsungen, Germany, D. Cosic: None declared, C. Schmoor: None declared, M. Dettenkofer Grant/Research support from B.Braun, Melsungen, Germany.

\section{Author details}

'Department of Environmental Health Sciences, University Medical Center Freiburg, Freiburg, Germany. ${ }^{2}$ Clinical Trials Unit, University Medical Center Freiburg, Freiburg, Germany.

Published: 29 June 2011

doi:10.1186/1753-6561-5-S6-P271

Cite this article as: Conrad et al: Safety and tolerability of virucidal

hand rubs: a randomized, double-blind, cross-over trial. BMC Proceedings 2011 5(Suppl 6):P271. 